\title{
Evaluation of Shelf Life of Processed Cheese by Implementing Neural Computing Models
}

\author{
Sumit Goyal, Gyanendra Kumar Goyal \\ National Dairy Research Institute, Karnal, India. \\ Email-thesumitgoyal@gmail.com,gkg5878@yahoo.com
}

\begin{abstract}
For predicting the shelf life of processed cheese stored at $7-8^{\circ} \mathrm{C}$, Elman single and multilayer models were developed and compared. The input variables used for developing the models were soluble nitrogen, $\mathrm{pH}$; standard plate count, Yeast \& mould count, and spore count, while output variable was sensory score. Mean Square Error, Root Mean Square Error, Coefficient of Determination and Nash - Sutcliffo Coefficient were applied in order to compare the prediction ability of the developed models. The Elman models got simulated very well and showed excellent agreement between the experimental data and the predicted values, suggesting that the EIman models can be used for predicting the shelf life of processed cheese.
\end{abstract}

Keywords- Artificial Neural Network, Artificial Intelligence, EIman, Processed Cheese, Shelf Life

\section{INTRODUCTION}

$\mathrm{A}^{\mathrm{n}}$ RTIFICIAL neural network (ANN), usually called neural network is a mathematical model or computational model that is inspired by the structure and functional aspects of ANN. ANN based computing method is an adaptive system that changes its structure based on external or internal information that flows through the network during the learning phase. In ANN based intelligent computing, simple artificial nodes called "neurons", "neurodes", "processing elements" or "units" are connected together to form a network of nodes mimicking the biological neural networks. Generally, ANN involves a network of simple processing elements that exhibit complex global behavior determined by connections between processing elements and element parameters. While an ANN does not have to be adaptive, its practical use comes with algorithms designed to alter the weights of the connections in the network to produce a desired signal flow [1].Elman models are two layered backpropagation networks, with the addition of a feedback connection from the output of the hidden layer to its input. This feedback path allows Elman model to learn to recognize and generate temporal patterns, as well as spatial patterns. The Elman ANN model has tansig neurons in its hidden layer, and purelin neurons in its output layer. This combination is special in that two layered networks with these transfer functions can approximate any function (with a finite number of discontinuities) with arbitrary accuracy. The only requirement is that the hidden layer must have enough neurons. More hidden neurons are needed as the function being fitted increases in complexity. Elman model differs from conventional two layer networks in that the first layer has a recurrent connection. The delay in this connection stores values from the previous time step, which can be used in the current time step. Therefore, even if two Elman models, with the same weights and biases, are given identical inputs at a given time step, their outputs can be different because of different feedback states. Because the network can store information for future reference, it is able to learn temporal patterns as well as spatial patterns. The Elman models can be trained to respond to, and to generate, both kinds of patterns [2].Shelf life studies can provide important information to product developers enabling them to ensure that the consumer will see a high quality product for a significant period of time after production. Of course, long shelf life studies do not fit with the speed requirement and therefore, accelerated studies have been developed as part of innovation [3]. Goyal and Goyal [4] implemented brain based artificially intelligent scientific computing models for shelf life detection of cakes stored at $30^{\circ} \mathrm{C}$. The potential of simulated neural networks for predicting shelf life of soft cakes stored at $10^{\circ} \mathrm{C}$ was highlighted by Goyal and Goyal [5]. Cascade single and double hidden layer models were developed for predicting the shelf life of Kalakand, a desiccated sweetened dairy product [6]. For forecasting the shelf life of instant coffee drink, radial basis artificial neural engineering and multiple linear regression models were suggested [7]. Cascade forward and feedforward backpropagation artificial intelligence models for prediction of sensory quality of instant coffee flavoured sterilized drink have been evolved [8]. Artificial neural networks for predicting the shelf life of milky white dessert jeweled with pistachio were applied by Goyal and Goyal [9]. The shelf life of brown milk cakes decorated with almonds was predicted by developing artificial neural network based radial basis (exact fit) and radial basis (fewer neurons) models [10]. Also, the time-delay and linear layer (design) intelligent computing expert system models have been developed for predicting the shelf life of soft mouth melting milk cakes stored at $6^{\circ} \mathrm{C}$ [11]. The computerized models have been 
suggested for predicting the shelf life of post-harvest coffee sterilized milk drink [12]. Neuron based artificial intelligent scientific computer engineering models estimated the shelf life of instant coffee sterilized drink [13]. The aim of the present study is to develop Elman ANN models with single layer and multilayer, and to compare them with each other, for predicting the shelf life of processed cheese stored at $7-8^{\circ} \mathrm{C}$.

\section{METHOD MATERIAL}

The input variables used in the network were the processed cheese experimental data relating to soluble nitrogen, $\mathrm{pH}$; standard plate count, Yeast \& mould count, and spore count. The sensory score assigned by the trained panelists was taken as output variable for developing computing models (Fig.1). Experimentally obtained 36 observations for each input and output variables were used for developing the models. The dataset was randomly divided into two disjoint subsets, namely, training set having 30 ( $80 \%$ for training) observations, and validation set (20\% for testing) consisting of 6 observations.

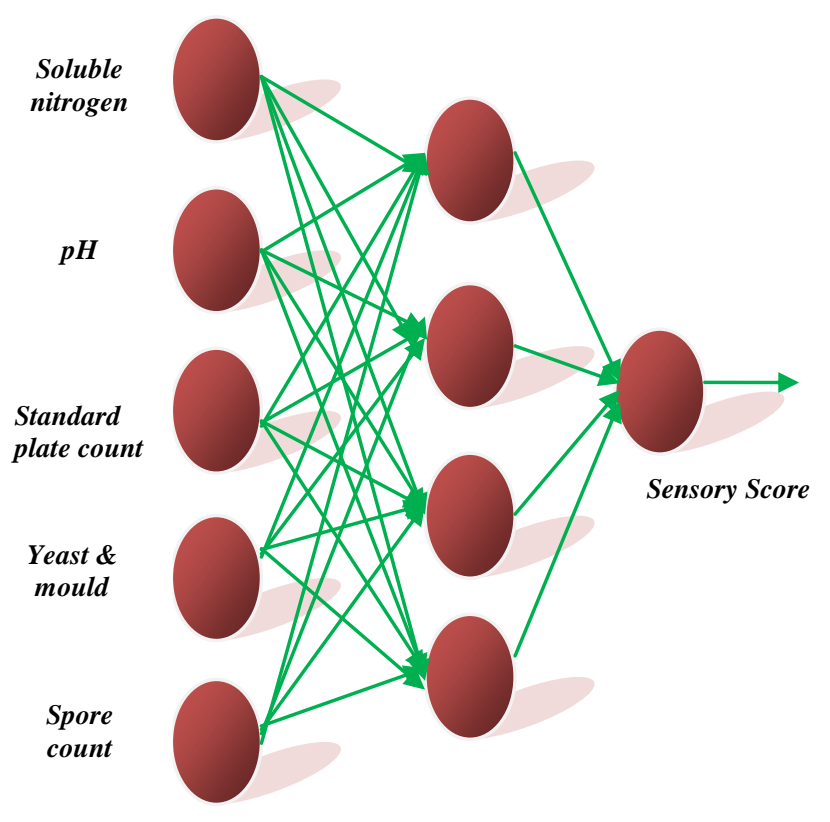

Fig. 1: Input and output parameters for elman models

$$
\begin{aligned}
M S E & =\left[\sum_{1}^{N}\left(\frac{Q_{\exp }-Q_{c a l}}{n}\right)^{2}\right]_{(1)} \\
R M S E & =\sqrt{\frac{1}{n}\left[\sum_{1}^{N}\left(\frac{Q_{\exp }-Q_{c a l}}{Q_{\exp }}\right)^{2}\right]}
\end{aligned}
$$

$$
\begin{aligned}
& R^{2}=1-\left[\sum_{1}^{N}\left(\frac{Q_{\exp }-Q_{c a l}}{Q_{\exp }{ }^{2}}\right)^{2}\right]_{(3)} \\
& E^{2}=1-\left[\sum _ { 1 } ^ { N } \left(\frac{Q_{\exp }-Q_{c a l}}{\left.\left.Q_{\exp }-\overline{Q_{\exp }}\right)^{2}\right]_{(4)}}\right.\right.
\end{aligned}
$$

Where,

$Q_{\text {exp }}=$ Observed value;

$Q_{\text {cal }}=$ Predicted value;

$\overline{Q_{\text {exp }}}=$ Mean predicted value;

$n=$ Number of observations in dataset.

Mean Square Error MSE (1), Root Mean Square Error RMSE (2), Coefficient of Determination $\mathrm{R}^{2}$ (3) and Nash - Sutcliffo Coefficient $\mathrm{E}^{2}$ (4) were applied in order to compare the prediction ability of the developed models. Gradient Descent algorithm with adaptive learning rate, Powell Beale restarts conjugate gradient algorithm, Levenberg Marquardt algorithm, Fletcher Reeves update conjugate gradient algorithm, and Bayesian regularization algorithms were tried. Bayesian regularization mechanism was finally selected for training ANN models, as it exhibited the best results. The network was trained up to 100 epochs, and neurons in each hidden layers varied from 1 to 20 . The network was trained with single as well as multiple hidden layers, and transfer function for hidden layer was tangent sigmoid, while for the output layer it was pure linear function. MALTAB software was used for performing experiments.

\section{RESULTS AND DISCUSSION}

Elman single layer (Table 1) and multilayer (Table 2) ANN models were developed and compared with each other for predicting the shelf life of processed cheese stored at $7-8^{\circ} \mathrm{C}$.

TABLE I

RESULTS FOR SINGLE LAYER ELMAN MODEL

\begin{tabular}{|c|c|c|c|c|}
\hline Neurons & $\boldsymbol{M S E}$ & $\boldsymbol{R M S E}$ & $\boldsymbol{R}^{\mathbf{2}}$ & $\boldsymbol{E}^{\mathbf{2}}$ \\
\hline 3 & $9.13178 \mathrm{E}-05$ & 0.009556034 & 0.990443966 & 0.999908682 \\
\hline 4 & 0.000314749 & 0.01774116 & 0.98225884 & 0.999685251 \\
\hline 5 & 0.000449704 & 0.021206231 & 0.978793769 & 0.999550296 \\
\hline 6 & $9.14141 \mathrm{E}-05$ & 0.009561074 & 0.990438926 & 0.999908586 \\
\hline 7 & 0.00039364 & 0.019840363 & 0.980159637 & 0.99960636 \\
\hline 8 & 0.00039364 & 0.019840363 & 0.980159637 & 0.99960636 \\
\hline 9 & $9.15588 \mathrm{E}-05$ & 0.009568634 & 0.990431366 & 0.999908441 \\
\hline 10 & $9.1607 \mathrm{E}-05$ & 0.009571154 & 0.990428846 & 0.999908393 \\
\hline
\end{tabular}




\begin{tabular}{|c|c|c|c|c|}
\hline 11 & 0.000330042 & 0.018167049 & 0.981832951 & 0.999669958 \\
\hline 12 & $3.34199 \mathrm{E}-05$ & 0.005780997 & 0.994219003 & 0.99996658 \\
\hline 13 & 0.000188492 & 0.013729239 & 0.986270761 & 0.999811508 \\
\hline 14 & $4.20792 \mathrm{E}-06$ & 0.002051322 & 0.997948678 & 0.999995792 \\
\hline 15 & $9.18484 \mathrm{E}-05$ & 0.009583755 & 0.990416245 & 0.999908152 \\
\hline 16 & $9.18967 \mathrm{E}-05$ & 0.009586275 & 0.990413725 & 0.999908103 \\
\hline 17 & $9.1945 \mathrm{E}-05$ & 0.009588795 & 0.990411205 & 0.999908055 \\
\hline 18 & 0.000115845 & 0.010763139 & 0.989236861 & 0.999884155 \\
\hline 19 & $9.20417 \mathrm{E}-05$ & 0.009593835 & 0.990406165 & 0.999907958 \\
\hline $\mathbf{2 0}$ & $\mathbf{1 . 8 7 8 7 8 E - 0 7}$ & $\mathbf{0 . 0 0 0 4 3 3 4 4 9}$ & $\mathbf{0 . 9 9 9 5 6 6 5 5 1}$ & $\mathbf{0 . 9 9 9 9 9 9 8 1 2}$ \\
\hline
\end{tabular}

TABLE 2

RESULTS FOR MULTILAYER ELMAN MODEL

\begin{tabular}{|c|c|c|c|c|}
\hline & & & & \\
Neurons & $\boldsymbol{M S E}$ & $\boldsymbol{R M S E}$ & $\boldsymbol{R}^{\mathbf{2}}$ & $\boldsymbol{E}^{\mathbf{2}}$ \\
\hline $3: 3$ & $9.1366 \mathrm{E}-05$ & 0.009558554 & 0.990441446 & 0.999908634 \\
\hline $4: 4$ & 0.000561383 & 0.023693521 & 0.976306479 & 0.999438617 \\
\hline $5: 5$ & $9.14141 \mathrm{E}-05$ & 0.009561074 & 0.990438926 & 0.999908586 \\
\hline $6: 6$ & $9.14141 \mathrm{E}-05$ & 0.009561074 & 0.990438926 & 0.999908586 \\
\hline $7: 7$ & $9.14623 \mathrm{E}-05$ & 0.009563594 & 0.990436406 & 0.999908538 \\
\hline $8: 8$ & $9.14623 \mathrm{E}-05$ & 0.009563594 & 0.990436406 & 0.999908538 \\
\hline $9: 9$ & $9.15105 \mathrm{E}-05$ & 0.009566114 & 0.990433886 & 0.999908489 \\
\hline $10: 10$ & $4.78872 \mathrm{E}-05$ & 0.006920061 & 0.993079939 & 0.999952113 \\
\hline $11: 11$ & 0.000535418 & 0.02313911 & 0.97686089 & 0.999464582 \\
\hline $12: 12$ & 0.000554478 & 0.023547358 & 0.976452642 & 0.999445522 \\
\hline $13: 13$ & $9.16552 \mathrm{E}-05$ & 0.009573674 & 0.990426326 & 0.999908345 \\
\hline $14: 14$ & $9.17035 \mathrm{E}-05$ & 0.009576194 & 0.990423806 & 0.999908296 \\
\hline $15: 15$ & $9.17518 \mathrm{E}-05$ & 0.009578715 & 0.990421285 & 0.999908248 \\
\hline $16: 16$ & $8.80247 \mathrm{E}-05$ & 0.009382151 & 0.990617849 & 0.999911975 \\
\hline $17: 17$ & $3.93431 \mathrm{E}-05$ & 0.006272407 & 0.993727593 & 0.999960657 \\
\hline $18: 18$ & $9.18484 \mathrm{E}-05$ & 0.009583755 & 0.990416245 & 0.999908152 \\
\hline $19: 19$ & 0.000711004 & 0.026664661 & 0.973335339 & 0.999288996 \\
\hline $\mathbf{2 0 : 2 0}$ & $\mathbf{1 . 1 7 9 8 1 E}-05$ & $\mathbf{0 . 0 0 3 4 3 4 8 3}$ & $\mathbf{0 . 9 9 6 5 6 5 1 7}$ & $\mathbf{0 . 9 9 9 9 8 8 2 0 2}$ \\
\hline
\end{tabular}

Elman single layer and multilayer computerized models were developed for predicting the shelf life of processed cheese stored at 7-8 C. Single layer model with 5-20-1 combination (MSE: 1.87878E-07; RMSE: 0.000433449; $\mathbf{R}^{2}$ : $\mathbf{0 . 9 9 9 5 6 6 5 5 1} ; \mathbf{E}^{\mathbf{2}}: \mathbf{0 . 9 9 9 9 9 9 8 1 2 )}$ gave the best result among single layer experiments (Table 1); and for multilayer Elman models, the best result was with 5-20-20-1 combination (MSE: 1.17981E-05; RMSE: 0.00343483; $\mathbf{R}^{2}: 0.99656517 ; \mathrm{E}^{2}$ : 0.999988202) (Table 2). The comparison of these two results showed that the multilayer model with a combination of 5-2020-1 performed better for predicting the shelf life of processed cheese. The comparison of Actual Sensory Score (ASS) and Predicted Sensory Score (PSS) for Elman single layer and multilayer models are illustrated in Fig.2 and Fig.3, respectively.

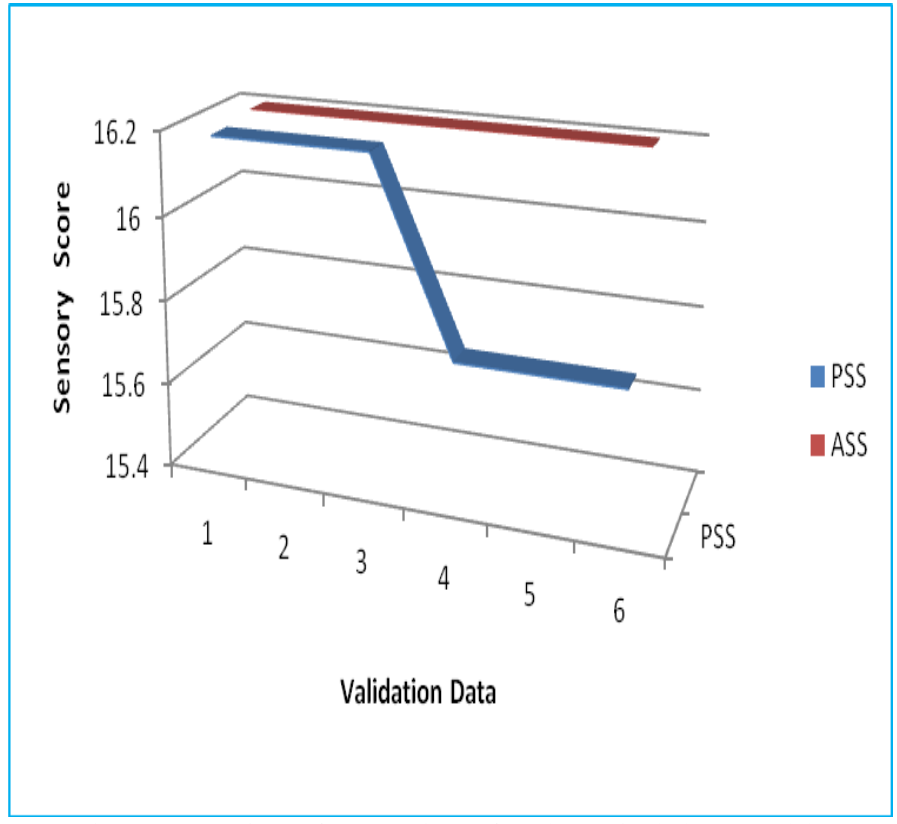

Fig. 2: Comparison of ASS and PSS for Elman single layer model

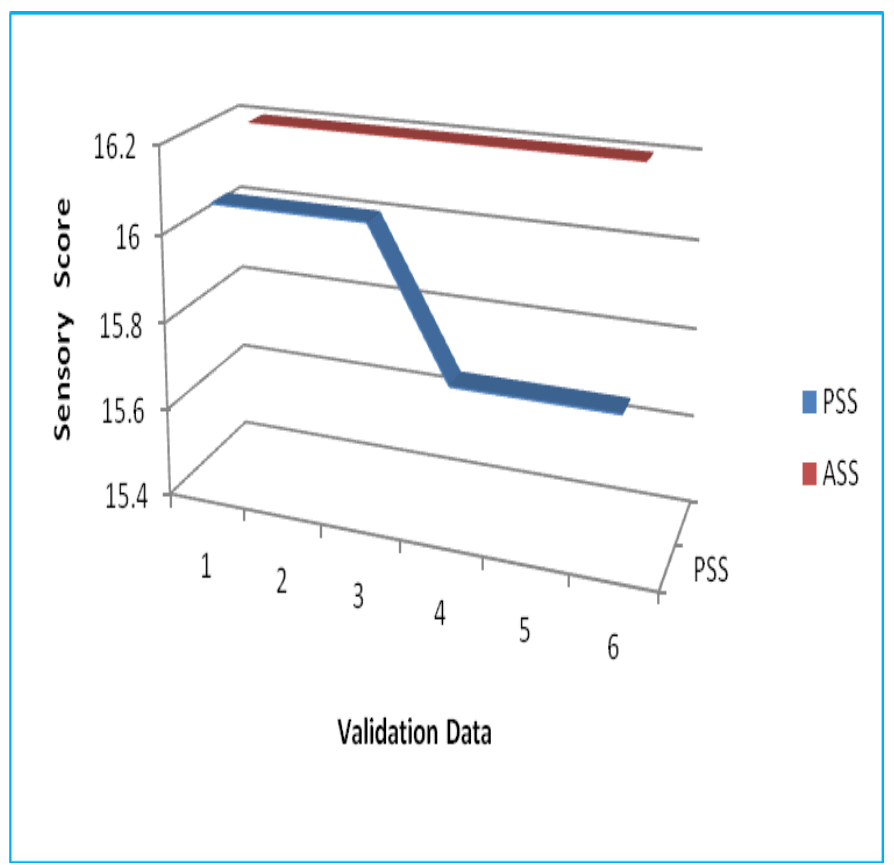

Fig. 3: Comparison of ASS and PSS for Elman multilayer model

From the results, it is observed that Elman models got simulated exceedingly well, and are very effective in predicting the shelf life of processed cheese stored at $7-8^{\circ} \mathrm{C}$.

\section{CONCLUSION}

Elman single and multilayer ANN models were developed and compared with each other. The inputs variables of the network consisted of soluble nitrogen, $\mathrm{pH}$; standard plate count, yeast $\&$ mould count, and spore count. The output variable was sensory score of the processed cheese stored at $7-8^{\circ} \mathrm{C}$. The modelling results revealed very good agreement between the 
experimental data and the predicted values, with a high determination coefficient, establishing that the developed Elman ANN models were able to analyze non-linear multivariate data with excellent performance, fewer parameters, and shorter calculation time. This Elman model might be an alternative low cost and less time consuming method for determining the expiration date of stored processed cheese, shown on labels and provide consumers with a safer food supply [14-20].

\section{V.REFERENCES}

[1] Artificial Nerural Network http://en.wikipedia.org/wiki/Artificial_neural_network (accessed on 4.7.2011)

[2] H. Demuth , M. Beale and M. Hagan. Neural network toolbox user's guide". The MathWorks Inc., Natrick, USA , (2009).

[3] www.medlabs.com/Downloads/food_product_shelf_life_web.pdf (accessed on 7.3.2011).

[4] Sumit Goyal and G.K. Goyal, "Brain based artificial neural network scientific computing models for shelf life prediction of cakes", Canadian Journal on Artificial Intelligence, Machine Learning and Pattern Recognition, 2(6), 73-77,2011.

[5] Sumit Goyal and G.K. Goyal, "Simulated neural network intelligent computing models for predicting shelf life of soft cakes", Global Journal of Computer Science and Technology, 11(14), Version 1.0, 29-33,2011.

[6] Sumit Goyal and G.K. Goyal, "Advanced computing research on cascade single and double hidden layers for detecting shelf life of kalakand: An artificial neural network approach", International Journal of Computer Science \& Emerging Technologies, 2(5), 292-295, 2011.

[7] Sumit Goyal and G.K. Goyal, "Application of artificial neural engineering and regression models for forecasting shelf life of instant coffee drink", International Journal of Computer Science Issues, 8(4), No 1, 320-324, 2011.

[8] Sumit Goyal and G.K. Goyal, "Cascade and feedforward backpropagation artificial neural networks models for prediction of sensory quality of instant coffee flavoured sterilized drink", Canadian Journal on Artificial Intelligence, Machine Learning and Pattern Recognition, 2(6), 78-82, 2011.

[9] Sumit Goyal and G.K. Goyal, "A new scientific approach of intelligent artificial neural network engineering for predicting shelf life of milky white dessert jeweled with pistachio", International Journal of Scientific and Engineering Research, 2(9), 1-4, 2011.

[10] Sumit Goyal and G.K. Goyal, "Radial basis artificial neural network computer engineering approach for predicting shelf life of brown milk cakes decorated with almonds", International Journal of Latest Trends in Computing, 2(3), 434-438, 2011.

[11] Sumit Goyal and G.K. Goyal, "Development of intelligent computing expert system models for shelf life prediction of soft mouth melting milk cakes", International Journal of Computer Applications, 25(9), 41-44, 2011.

[12] Sumit Goyal and G.K. Goyal, "Computerized models for shelf life prediction of post-harvest coffee sterilized milk drink", Libyan Agriculture Research Center Journal International, 2 (6), 274-278, 2011.

[13] Sumit Goyal and G.K. Goyal, "Development of neuron based artificial intelligent scientific computer engineering models for estimating shelf life of instant coffee sterilized drink", International Journal of Computational Intelligence and Information Security, 2(7), 4-12,2011.

[14] Sumit Goyal and G.K. Goyal, "Soft computing single hidden layer models for shelf life prediction of burfi", Russian Journal of Agricultural and Socio-Economic Sciences, 5(5), 28-32, 2012.

[15] Sumit Goyal and G.K. Goyal, "Predicting shelf life of dairy product by using artificial neural networks (ANN) and statistical computerized methods", International Journal of Computer Engineering Research, 3(2), 20-24, 2012.

[16] Sumit Goyal and G.K. Goyal, "Time - delay single layer artificial neural network models for estimating shelf life of burfi", International Journal of Research Studies in Computing, 1(2), 11-18, 2012.
[17] Sumit Goyal and G.K. Goyal, "Study on single and double hidden layers of cascade artificial neural intelligence neurocomputing models for predicting sensory quality of roasted coffee flavoured sterilized drink", International Journal of Applied Information Systems, 1(3), 1-4, 2012.

[18] Sumit Goyal and G.K. Goyal, "Shelf life determination of kalakand using soft computing technique", Advances in Computational Mathematics and its Applications, 1(3), 131-135, 2012.

[19] Sumit Goyal and G.K. Goyal, "Radial basis (exact fit) and linear layer (design) ANN models for shelf life prediction of processed cheese", International Journal of $\mathrm{u}-$ and e- Service, Science and Technology, 5(1), 63-69, 2012

[20] Sumit Goyal and G.K. Goyal, "Central nervous system based computing models for shelf life prediction of soft mouth melting milk cakes", International Journal of Information Technology and Computer Science, 4(4), 33-39, 2012.

\section{Author's Biodata}

Sumit Goyal: is M.Phil. in Computer Science, Master of Computer Applications and Bachelor of Information Technology. His research interests have been in the area of soft computing, artificial neural networks and prediction of shelf life of food products. His research has appeared in Canadian Journal on Artificial Intelligence, Machine Learning and Pattern Recognition, Int. J. of Computer Applications, Int. J. of Computational Intelligence and Information Security, Int. J. of Latest Trends in Computing, Int. J. of Scientific and Engineering Research, Int. J. of Computer Science Issues, Int. J. of Computer Science \& Emerging Technologies, Global Journal of Computer Science and Technology, Int. J. of Artificial Intelligence and Knowledge Discovery, amongst others. He is member of IDA.

Gyanendra Kumar Goyal: obtained his Ph.D. degree in 1979 from Panjab University, Chandigarh, India. He was recipient of United Nations fellowship award and World Bank's fellowship award. He was also awarded Belgian Government's fellowship award. In 198586, he did specialized research work on Dairy and Food Packaging at Michigan State University, East Lansing, U.S.A.; and in the year 1999 he received advanced training in Education Technology at Cornell University, New York, U.S.A. His research interests include dairy \& food packaging and shelf life determination of food products. $\mathrm{He}$ has published more than 150 research papers in national and international journals, and presented his work in national and international conferences. His research work has been published in Int. J. of Food Sci. Technol. and Nutrition, Nutrition and Food Science, Milchwissenschaft, American Journal of Food Technology, British Food Journal, Canadian Journal on Artificial Intelligence, Machine Learning and Pattern Recognition, Int. J. of Computer Applications, Int. J. of Computer Science Issues, Int. J. of Computer Science \& Emerging Technologies, Int. J. of Artificial Intelligence and Knowledge Discovery, amongst others. He is life member of AFST (I) and IDA. 